\title{
Raluca BUTUROIU* \\ Thoughts on Antisocial Media. How Facebook Disconnects Us and Undermines Democracy by Siva Vaidhyanathan
}

The impact of social media on public affairs tends to be discussed with reference to specific contexts. Two prominent examples are the British referendum campaign in June 2016, and the successful candidacy of Donald Trump, concluded that same year in November. Recent research looking at such events identify them as clear examples of how social media "have transformed our communication, how we access, and engage with information" (Hänska \& Bauchowitz, 2017, p. 27). The two particular unanticipated outcomes referred to above - among others - attracted numerous scholars in the attempt to find possible explanations. Currently, scholars from fields such as communication studies, political science, and tech studies have started to focus on unveiling potential negative effects of social media on society. Given the magnitude of events such as the Cambridge Analytica scandal in early 2018, after which Facebook usage has collapsed (Hern, 2019), this was to be expected.

Research interests in this area have revolved around illuminating the dark side of social networking sites (especially Facebook and Twitter). And the light thrown has at times been quite harsh. Here are a few examples of how Facebook has been being portrayed since 2016: "the biggest threat to our democracy", as Cambridge Analytica whistleblower Brittany Kaiser ${ }^{1}$ says; "a digital gangster destroying democracy" (Cadwalladr, 2019); a tool which is "rotting democracy from within" (DemDigest, 2019); a "machine" which "disconnects us and undermines democracy". The latter are Siva Vaidhyanathan's words, and they make for a telling introduction to the analysis he develops in his 2018 book Antisocial Media. How Facebook Disconnects Us and Undermines Democracy.

Vaidhyanathan's book is an ample response to "Facebook's toxicity", largely ignored by both experts and non-experts as recently as 2015 and 2016. The massive ignorance regarding the effects of Facebook could be associated with the fact that people had

\begin{abstract}
grown so accustomed to Facebook's effects that they stopped thinking about them entirely. Facebook disappeared from their consciousness. It happened in much the same way we don't think about the hum of our refrigerators, or central heating, or highways. Facebook was just there - a part of life. Facebook, however, differs from physical infrastructure in one important way: it doesn't occupy your kitchen or basement. Facebook occupies your brain (Horgan, 2018).
\end{abstract}

${ }^{*}$ National University of Political Science and Public Administration, Bucharest, Romania raluca.buturoiu@comunicare.ro 
It is in a timely book that this verdict develops into an argument, one which fits well into a socio-political paradigm in which "manipulation of our media environment by foreign as well as domestic actors is now the new normal" (Naughton, 2018). Probably guided by the relative weight of the networking sites, Vaidhyanathan highlights the potential dangers associated with the one that is currently used by most people, Facebook. While the topic is not novel, in my view, the novelty and significant contribution of the book resides in its critical perspective, which allows for personal reflection and interpretation.

A wide angle lens would capture the book as an attempt to offer a critical picture on Facebook's effects at the societal level. And this is a perspective which is rather absent from the literature to date on social media effects. There is a large variety of studies focusing on individual-level effects (e.g. on Facebook effects on loneliness, self-esteem, life satisfaction, selfreported depression, body image, etc.), while the "bird's eye-view" effects (i.e., the effects at the societal level) have been comparatively rarely addressed. Continuing Neil Postman's reflexive approach about another very pervasive mass communication medium - television Siva Vaidhyanathan offers insights on how Facebook, as the most pervasive element within the current media ecosystem, can change the structure of communication "by creating new forms of truth-telling" (Silvestre, 2018).

My thoughts on the book were fueled mainly by my previous experience with media and communication research studies. Given this background, in my opinion, the book might be interpreted in the perspective of the currently dominant paradigm of media effects research: preference-based effects models. This paradigm combines elements from both weak and strong media effects research, while focusing on three characteristics that could explain the power of a media message: (1) narrowcasting information towards ideologically fragmented individuals, (2) a tendency among individuals to rely on self-selected information - often labeled echo chambers or filter bubbles, and (3) personalized news aggregators leading to narrower and narrower information tailoring based on both voluntary and involuntary user input (Cacciatore, Scheufele, \& Iyengar, 2016, p. 19).

Within this recent paradigm, the author presents seven "machines" responsible for the potential harmful effects of social media. The pleasure machine is the concept used to refer to the array of sentiments associated with Facebook use - including anxiety, anger, and resentment. The pleasure associated with Facebook is what makes people come back or keeps them permanently connected. In fact, it is not real pleasure, but a combination of habit and addiction, which turns out to be rather noxious. There is then the surveillance machine - the description the author uses to characterize how Facebook is working within the current digital ecosystem, with strong emphasis on the ideas of privacy and dignity, which seem to be left behind. Vaidhyanathan continues with the attention machine - mainly the mechanism through which Facebook keeps people on the platform and makes them coming back. In this case, "attention feeds thought" (2018, p. 80) is the idea which might explain why some beliefs turn into attitudes and, then, into possible behaviors.

The benevolence machine explains the very beginning of Facebook and the philosophy behind its creation and development - it was initially created as a platform which gave voice to ordinary people, in the attempt to create a better world. A related idea is the protest machine, which exemplifies how Facebook and other social media networks play a role in social and political movements, especially in terms of making people more aware or sensitive to particular issues. The politics machine refers explicitly to the way Facebook is used for political purposes; tailored persuasion is referred to as one of the most successful ways of tar- 
geting political messages in order to obtain certain outcomes - by manipulating and targeting information or propaganda. On a darker take, the disinformation machine is the concept Vaidhyanathan uses to describe the way Facebook is used "to undermine trust in the institutions that make democracy function, or to disrupt democratic deliberation" (2018, p. 180).

After disassembling these "machines" through which Facebook performs its work of disconnecting people and undermining democracy, the author suggests another diagnostic label for Facebook, namely the nonsense machine. It captures the claim that, when Facebook dominates people's sense of the world, they "become carriers of extremist nonsense" (Vaidhyanathan, 2018, p. 6), and, in my view, it is perhaps the strongest warning this book delivers.

The machines or machineries through which Facebook dominates whole populations, and in a sense the entire world, are presented in a friendly, yet very alarmist manner. The urgency, I think, is quite clear. We find ourselves in a context in which it is imperative, first, that we become aware of the challenges facing us, and then try to arrive at some solutions. Nevertheless, it is not as clear in the book who is, or better said, who should be held responsible for the chaos, pollution, or damage caused by the present media ecosystem.

Before going further with some thoughts about possible implications of the book, in the following lines I will refer to the concepts of privacy and targeting. Both of them are comprehensively discussed in this volume, and should be of importance for the expert and lay reader alike. Moreover, the author stresses the value of privacy in a memorable manner. Here is an example:

Privacy is not a substance we can trade away. It's not a state of being that either exists or does not exist. And it can't “die", as too many people have declared. Privacy is a power we exercise - or hope to exercise - when we see fit. (Vaidhyanathan, 2018, p. 73).

Privacy is regarded as a "power"; indeed, only those who have enough power can afford keeping their lives away from Facebook. But how easy is to regain this power? Once online, it can be difficult to separate private and public life, online and offline selves (Burkell, Fortier, Wong, \& Simpson, 2014; Ford, 2011). The main problem associated with privacy is who assumes responsibility for it. Here, the question is how individual is the privacy of each person as this is no longer a matter to be settled at individual level? And who truly succeeds in maintaining control over her privacy? A person who does not reveal personal details online? Or maybe one who gives false information about herself? Or only one who does not have a Facebook account?

Another important point tackled in the book is targeted or personalized persuasion. The evolution and large scale usage of social networks have brought important changes within the process of persuasion. The answer to the questions related to how difficult or easy is to persuade people with a media message nowadays is very provocative. One the one hand, it might seem easy to get access to people, and thus to persuade them; the multitude of communication channels and the instant reach through which they can be targeted seem to help. Furthermore, research in the areas of persuasion and propaganda is growing and people who want to create a persuasive message (no matter its goal) can benefit from it. On the other hand, it might seem more difficult to persuade someone mainly because of the wide access to information; it is very easy nowadays to get access to different sources of information (including opposing viewpoints about certain issues) and, as a consequence, to build less biased opinions and attitudes.

These provisos aside (they can be quite rational and predictable), one of the most important challenges brought by the evolution and acceleration of digital technologies is the rise 
of computational propaganda - targeted messages distributed to certain groups of individuals, according to their personal characteristics and prior media exposure/ experience. The impact and effects of personalized media messages have been intensively studied during the last years (Hirsh, Kang, \& Bodenhausen, 2012; Luong, Garrett, \& Slater, 2019; Oyibo \& Vassileva, 2019). Specifically, the outcomes of the US election and of the UK referendum in 2016, as well as the rise of "illiberal democracy" discourse mainly in Central and Eastern European countries, have attracted researchers from both political and media effects studies. Consequently, the academic literature on topics related to the influence and effects of social media (especially Facebook and Twitter) is impregnated with concepts such as enhanced selectivity of the audience, ideologically-driven news, confirmation bias, filter bubbles, echo chambers - all being related to some extent to the umbrella concept of tailored or personalized persuasion (Kushin, Yamamoto, \& Dalisay, 2019; Park, Jang, Lee, \& Yang, 2018; Spohr, 2017). The following is typical:

One unique attribute of social media is enhanced selectivity. Social media afford users the ability to selectively interact with like-minded individuals and seek ideologically congruent opinions, which might contribute to forming echo chambers or filter bubbles where people attend largely to ideologically aligned content while shutting off cross-cutting opinions (Kushin, Yamamoto, \& Dalisay, 2019, p. 2).

These concepts - most of which are also present in Vaidhyanathan's book - are of particular importance as they define certain aspects of the current media environment. However, they are rarely used in order to find strategies and solutions to the issues to which they refer. For example, in my view, these concepts could be used in order to define and design a strategy of media literacy (i.e. a strategy which could include both technical/technological development, such as fact checking techniques, but also some actual actions which could improve some personal skills, such as critical thinking or rational rather than emotional responses to some online issues) (Jones-Jang, Mortensen, \& Liu, 2019).

One may have expected in this book, too, the discussion about the harmful effects of Facebook at the societal level to be followed by references to possible hints or solutions to be used by common people. In this sense, the diagnosis section of the book could be expanded with a prognosis one - maybe referring to the possibility of finding individual solutions to this societal issue, which the author successfully manages to describe, discuss and contextualize. Is closing down the platform the only viable (however improbable) solution?

Furthermore, it would be interesting to discuss the effects of social media (and Facebook in particular) in numbers. Specifically, within the book the impact of Facebook was mainly qualified, and not so much quantified. Again, with reference to what should be done to overcome the present "garbage" (Vaidhyanathan, 2018, p. 11) which is now on Facebook, a picture painted in quantitative terms may be a useful guide. And maybe it could also be a good idea to start with more accurate measurings of effects (at a societal level) in order to identify possible solutions.

All in all, Antisocial Media. How Facebook Disconnects Us and Undermines Democra$c y$ is a very well-documented book offering a version of the big picture of the (potential) negative effects of Facebook on communities and whole societies. By discussing the main areas where Facebook operates within society, the author puts together a comprehensive perspective about two important issues regarding Facebook, namely "how it works and how people use it" (Vaidhyanathan, 2018, p. 10). This is useful for both specialized researchers and the general readership. Moreover, by signaling the main negative effects of Facebook on democracy, the book contributes to the cultivation of public awareness towards how our opinions, attitudes, and behaviors are shaped in the present media ecosystem. 


\section{Note}

${ }^{1}$ https://www.cnbc.com/2019/10/22/brittany-kaiser-facebook-is-the-biggest-threat-to-us-democracy.html

\section{References}

Burkell, J., Fortier, A., Wong, L. (Lola) Y. C., \& Simpson, J. L. (2014). Facebook: public space, or private space? Information, Communication \& Society, 17(8), 974-985.

Cacciatore, M. A., Scheufele, D. A., \& Iyengar, S. (2016). The End of Framing as we Know it ... and the Future of Media Effects. Mass Communication and Society, 19(1), 7-23.

Cadwalladr, C. (2019, February 18). A digital gangster destroying democracy: the damning verdict on Facebook. The Guardian. Retrieved from https://www.theguardian.com/technology/2019/feb/18/a-digitalgangster-destroying-democracy-the-damning-verdict-on-facebook.

Ford, S. M. (2011). Reconceptualizing the public/ private distinction in the age of information technology. Information, Communication \& Society, 14(4), 550-567.

Hänska, M. \& Bauchowitz, S. (2017). "Tweeting for Brexit: how social media influenced the referendum". In J. Mair, Clark, T., Fowler, N., Snoddy, R., \& Tait, R. (eds.). Brexit, Trump and the Media. Retrieved from http://eprints.lse.ac.uk/84614/1/Hanska-Ahy_tweeting-for-brexit.pdf.

Hern, A. (2019, June 20). Facebook usage falling after privacy scandals, data suggests. The Guardian. Retrieved from https://www.theguardian.com/technology/2019/jun/20/facebook-usage-collapsed-since-scandal-data-shows.

Hirsh, J. B., Kang, S. K., \& Bodenhausen, G. V. (2012). Personalized persuasion: Tailoring persuasive appeals to recipients' personality traits. Psychological science, 23(6), 578-581.

Horgan, C. (2018, November 16). Facebook Is Now Critical to Democracy. That's a Problem. Medium. Retrieved from https://medium.com/s/story/facebook-is-now-critical-to-democracy-thats-a-problem$9493767442 b 1$.

Jones-Jang, S. M., Mortensen, T., \& Liu, J. (2019). Does Media Literacy Help Identification of Fake News? Information Literacy Helps, but Other Literacies Don't. American Behavioral Scientist, 1-18.

Kushin, M. J., Yamamoto, M., \& Dalisay, F. (2019, April-June). Societal Majority, Facebook, and the Spiral of Silence in the 2016 US Presidential Election. Social Media + Society, 1-12.

Luong, K. T., Garrett, R. K., \& Slater, M. D. (2019). Promoting Persuasion With Ideologically Tailored Science Messages: A Novel Approach to Research on Emphasis Framing. Science Communication, 41(4), $488-515$.

Naughton, J. (2018, December 23). Social media is an existential threat to our idea of democracy. Retrieved from https://www.theguardian.com/commentisfree/2018/dec/23/social-media-existential-threat-ideademocracy.

Oyibo, K., \& Vassileva, J. (2019). The relationship between personality traits and susceptibility to social influence. Computers in Human Behavior, 98, 174-188.

Park, Y. J., Jang, S. M., Lee, H., \& Yang, G. S. (2018, July-September). Divide in Ferguson: Social Media, Social Context, and Division. Social Media + Society, 1-13.

Silvestre, D. (2018, September, 11). Lessons from Amusing Ourselves to Death by Neil Postman. Medium. Retrieved from https://medium.com/@dsilvestre/lessons-from-amusing-ourselves-to-death-by-neil-postman-962221ee622.

Spohr, D. (2017). Fake news and ideological polarization: Filter bubbles and selective exposure on social media. Business Information Review, 34(3), 150-160.

*** Are social media platforms 'rotting democracy from within'? (2019, January 22). DemDigest. Retrieved from https://www.demdigest.org/are-social-media-platforms-rotting-democracy-from-within/. 